\title{
The Balloon Array for RBSP Relativistic Electron Losses (BARREL)
}

\author{
R.M. Millan • M.P. McCarthy · J.G. Sample • D.M. Smith • L.D. Thompson • \\ D.G. McGaw · L.A. Woodger · J.G. Hewitt · M.D. Comess · K.B. Yando • A.X. Liang • \\ B.A. Anderson · N.R. Knezek · W.Z. Rexroad • J.M. Scheiman • G.S. Bowers • \\ A.J. Halford · A.B. Collier • M.A. Clilverd • R.P. Lin • M.K. Hudson
}

Received: 24 March 2012 / Accepted: 22 December 2012 / Published online: 26 March 2013

(C) The Author(s) 2013. This article is published with open access at Springerlink.com

Abstract BARREL is a multiple-balloon investigation designed to study electron losses from Earth's Radiation Belts. Selected as a NASA Living with a Star Mission of Opportu-

R.M. Millan (凶) · D.G. McGaw · L.A. Woodger · B.A. Anderson · N.R. Knezek · J.M. Scheiman ·

A.J. Halford · M.K. Hudson

Dept. of Physics and Astronomy, Dartmouth College, Hanover, NH 03755, USA

e-mail: Robyn.Millan@dartmouth.edu

M.P. McCarthy

Dept. of Earth and Space Sciences, University of Washington, Seattle WA 98195-1310, USA

J.G. Sample · R.P. Lin

Physics Department and Space Sciences Laboratory, University of California at Berkeley, Berkeley, CA 94720, USA

D.M. Smith · A.X. Liang · W.Z. Rexroad · G.S. Bowers

Dept. of Physics, University of California, Santa Cruz, CA 95064, USA

L.D. Thompson

NASA Wallops Flight Facility, Wallops Island, VA 23337, USA

J.G. Hewitt

Department of Physics (DFP), USAF Academy, Colorado Springs, CO 80840, USA

M.D. Comess

Space X, Hawthorne, CA 90250, USA

K.B. Yando

Space Sciences Laboratory, University of California at Berkeley, Berkeley, CA 94720, USA

A.B. Collier
School of Physics, University of Kwazulu-Natal, Durban 4001, South Africa

M.A. Clilverd

British Antarctic Survey, Cambridge CB3 0ET, UK

R.P. Lin

School of Space Research, Kyung Hee University, Yongin, Korea 
nity, BARREL augments the Radiation Belt Storm Probes mission by providing measurements of relativistic electron precipitation with a pair of Antarctic balloon campaigns that will be conducted during the Austral summers (January-February) of 2013 and 2014. During each campaign, a total of 20 small $(\sim 20 \mathrm{~kg})$ stratospheric balloons will be successively launched to maintain an array of $\sim 5$ payloads spread across $\sim 6$ hours of magnetic local time in the region that magnetically maps to the radiation belts. Each balloon carries an $\mathrm{X}$-ray spectrometer to measure the bremsstrahlung X-rays produced by precipitating relativistic electrons as they collide with neutrals in the atmosphere, and a DC magnetometer to measure ULF-timescale variations of the magnetic field. BARREL will provide the first balloon measurements of relativistic electron precipitation while comprehensive in situ measurements of both plasma waves and energetic particles are available, and will characterize the spatial scale of precipitation at relativistic energies. All data and analysis software will be made freely available to the scientific community.

Keywords Radiation belts · Wave-particle interactions · Electron precipitation

\section{Introduction}

The loss of radiation belt particles has long been attributed to scattering into Earth's atmosphere (e.g., Walt and MacDonald 1962). Losses help define the structure of the radiation belts, for example, giving rise to the characteristic slot region between the inner and outer electron zones (Lyons and Thorne 1973). Precipitation into the atmosphere can be significant, completely depleting the radiation belts of electrons during the main phase of some geomagnetic storms (e.g., O’Brien et al. 2004; Selesnick 2006). Such rapid loss places a major constraint on the acceleration rate required to re-populate the belts on the timescale of a few days. Quantifying the electron loss rate is thus critically important for understanding the acceleration processes acting in the belts. Recently, loss to the magnetopause was shown to also be significant, particularly during storms (Ukhorskiy et al. 2006; Ohtani et al. 2009; Turner et al. 2012). The relative importance of magnetopause and atmospheric loss is currently unknown.

Wave-particle interactions are believed to be the main cause of scattering into the atmospheric loss cone (see e.g., Millan and Thorne 2007 and Thorne 2010 for a review). However, there is still very little quantitative comparison between theoretical predictions and observational data. The recent discovery of very large-amplitude whistler-mode waves in the magnetosphere (Cattell et al. 2008) raises questions about the validity of the quasi-linear treatment of wave-particle interactions that is typical of diffusive radiation belt models. It is important to establish when and where this approach is applicable.

The primary objective of BARREL is to understand relativistic electron precipitation. BARREL measures precipitation using an array of stratospheric balloons located in the region that magnetically maps to the radiation belts. Simultaneous measurements of precipitation by multiple balloon payloads distributed in $L$-value and magnetic local time allow BARREL to measure the electron loss rate across the outer zone, and probe the large-scale spatial structure of high energy precipitation. BARREL will augment the RBSP mission by providing measurements of precipitation which are difficult to obtain from an equatorial platform due to the small size of the equatorial loss cone. Conjunctions between the BARREL payloads and RBSP spacecraft will allow for direct, quantitative tests of theoretical models of precipitation by wave-particle interactions. BARREL is also complementary to other LEO assets that measure precipitation such as the NOAA-POES spacecraft. The 
Table 1 BARREL investigation specifications

\begin{tabular}{ll}
\hline Attribute & Value \\
\hline Payload Mass & $24 \mathrm{~kg}$ \\
Average Payload Power & $4.3 \mathrm{~W}$ \\
Payload Telemetry Rate & $2.1 \mathrm{kbits} / \mathrm{s}$ \\
Expected Campaign Durations & $1 / 1 / 13-2 / 10 / 13 ; 1 / 1 / 14-2 / 10 / 14$ \\
Expected Longitude Coverage & $0^{\circ}-150^{\circ} \mathrm{W}$ \\
Expected $L$-value Coverage & $3-7$ \\
\hline
\end{tabular}

balloon-based BARREL platform is nearly stationary, allowing for separation of temporal and spatial variations.

A major challenge of BARREL is the manufacture of 45 identical payloads ( 20 for each campaign and 5 spares) using the resources found at a typical research University. In this sense, BARREL is a pathfinder for future multi-spacecraft space physics missions. Critical to this effort was the production of multiple prototype payloads. This allowed for validation of the payload design, but also for refinement of the procedures for tracking, integrating, and testing payloads in parallel. Improvements to the design necessary for streamlining production were also made possible by building and testing early prototypes of the payload and instruments. Test campaigns, conducted from McMurdo, Antarctica in 2009 and 2010 with support from the NSF and NASA/CSBF, allowed for validation of the payload design in the actual flight environment, and improvement of the operations plans. Science data were also obtained and preliminary results are summarized in Millan (2011).

This paper provides a comprehensive overview of the BARREL investigation. Table 1 gives an overview of the BARREL payload and campaign specifications. In Sect. 2, we provide a more detailed discussion of the science objectives and discuss some mission design considerations for obtaining science closure. BARREL takes advantage of the instrument development done for the NSF-funded MINIS 2005 balloon campaign (Kokorowski et al. 2008). The instrumentation and support systems, described in Sects. 3 and 4 respectively, are largely based on MINIS. Instrument calibration is described in Sect. 5, and BARREL data products and data analysis are described in Sect. 6.

\section{Science Objectives and Design Considerations}

\subsection{Types of Relativistic Electron Precipitation}

At least two different types of relativistic electron precipitation have been reported and are thought to be caused by different mechanisms. The two types are differentiated by their temporal profiles and distribution in magnetic local time as described in detail in Millan (2011), and briefly reviewed here.

Rapid ( $\sim 250 \mathrm{~ms}$ ), intense bursts of precipitating electrons, called "microbursts" were first observed in the 10-100 keV energy range using balloon-based instrumentation (Anderson and Milton 1964). Microbursts were later shown to extend to relativistic energies (Imhof et al. 1992), and studied extensively with data from the SAMPEX satellite (e.g., Blake et al. 1996; Nakamura et al. 2000; O'Brien et al. 2004). The top panel of Fig. 1 shows an example of microburst precipitation detected by the MAXIS balloon payload during a geomagnetic storm in January 2000. The occurrence of microbursts peaks between $L=4-6$ on the dawnside and during geomagnetic storms, similar to the distribution of whistler-mode 

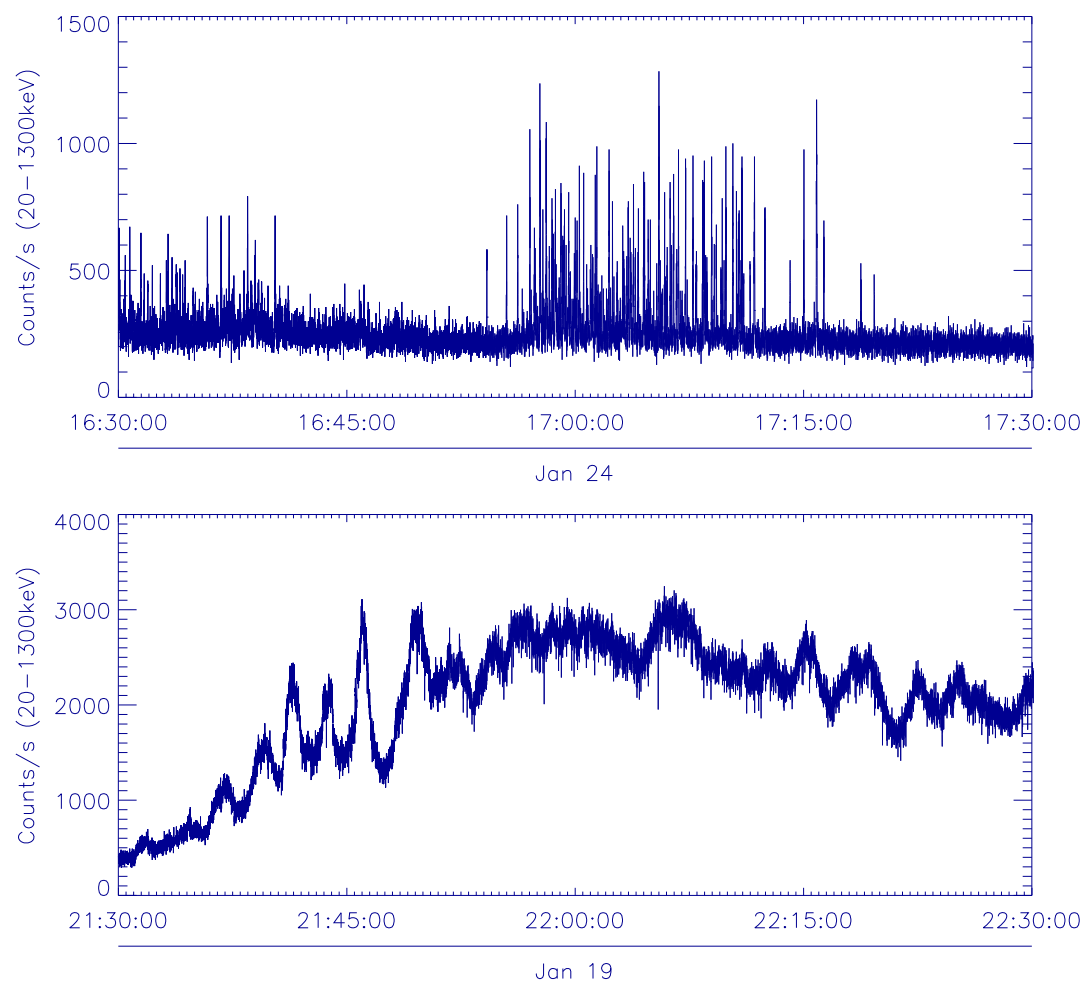

Fig. 1 Microburst precipitation (top) and duskside precipitation (bottom) observed by the MAXIS balloon experiment on January 24 and January 19, 2000 respectively. Both panels extend over a one hour time interval

chorus (e.g., Lorentzen et al. 2001b). The loss rate due to microbursts was estimated to be fast enough to empty the outer zone in about one day (O'Brien et al. 2004), but this analysis relied on an estimate for the spatial scale of the microburst region based on a statistical distribution. Refinement of this estimate will require knowledge of the instantaneous extent of the microburst precipitation region.

On the duskside, relativistic precipitation occurs with slower time variations, from minutes to hours (Millan et al. 2002). Unlike microbursts, duskside precipitation is not strongly correlated with geomagnetic storms and is known to occur over a range of magnetic activity levels, from small, isolated substorms (Lorentzen et al. 2000) to major CME-driven storms (Kokorowski et al. 2008). The occurrence has a broad radial distribution ranging from $L=3-8$. Duskside events are often modulated at ULF timescales (e.g., Millan et al. 2007), evident in the bottom panel of Fig. 1 which shows an example of duskside precipitation measured by the MAXIS balloon payload. Pitch-angle scattering by electromagnetic ion cyclotron (EMIC) waves, proposed much earlier on theoretical grounds (Thorne and Kennel 1971), may be the precipitation mechanisms (e.g., Foat et al. 1998; Lorentzen et al. 2000). However, this hypothesis is based primarily on the duskside location, and simultaneous measurements of the waves and precipitation have so far not been available to test it.

Recent work using data from SAMPEX suggests that duskside precipitation dominates over microburst loss at energies above $1 \mathrm{MeV}$, but that a third type of precipitation that is not localized in local time and does not belong to the two types described above appears to 
Fig. 2 Schematic of BARREL over Antarctica showing rough locations of SANAE IV (red diamond), Halley VI (green diamond), South Pole (blue diamond), the geomagnetic pole (cyan dot) and example balloon locations (white balloons). The blue dashed line represents $70^{\circ} \mathrm{S}$ geographic latitude, and the cyan dashed lines are lines of constant geomagnetic latitude at $50^{\circ}, 60^{\circ}$, $70^{\circ}$, and $80^{\circ}$

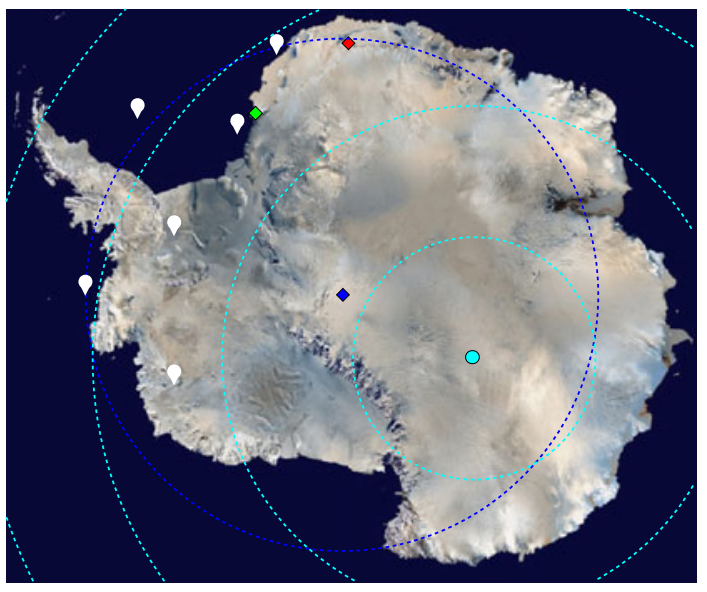

Fig. 3 Balloon trajectory in IGRF $L$-value assuming constant geographic latitude of $-71.7^{\circ}$ (solid thin line) along with three real trajectories from the MINIS 2005 balloon campaign (thick red, blue, and green lines). The payload speed is based on MINIS but can be significantly faster. For example, in January 2000, the MAXIS balloon moved about three times as fast at a similar geographic latitude $\left(-74^{\circ}\right)$

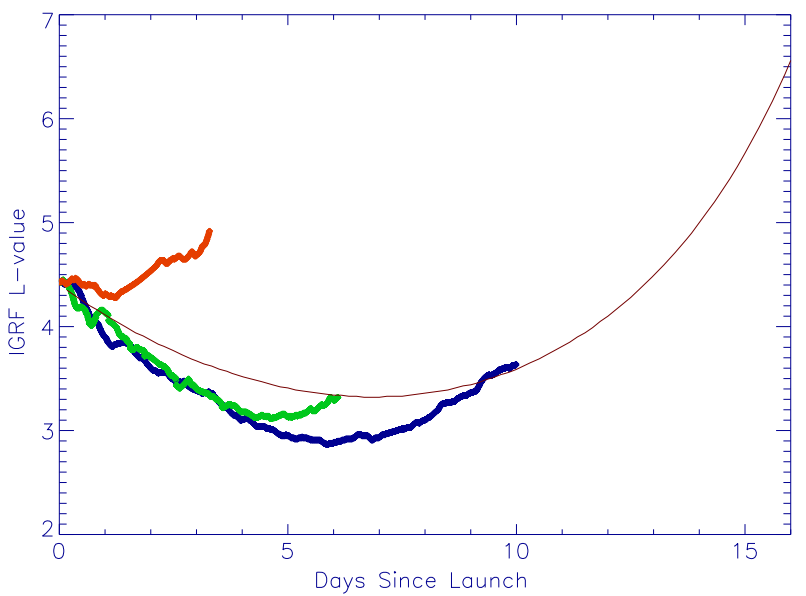

dominate the overall loss rate, possibly because it represents a constant, low flux "drizzle" (D.M. Smith private comm.). Though further work is required to confirm these preliminary results, it is clear that neither the importance nor the mechanism responsible for relativistic electron precipitation are well understood.

BARREL is designed to measure all types of electron precipitation between $100 \mathrm{keV}$ and a few MeV. To detect microbursts, measurements will be made with $50 \mathrm{~ms}$ time resolution. The balloon array will extend across $L$-values ranging from 3-7, allowing for measurements of precipitation across the outer zone. This is achieved with long duration balloons (LDBs) launched in the polar regions during summer. The circumpolar vortex winds carry each balloon westward away from the launch sites, allowing the array to be established after a few successive launches during the first week of the campaign (Fig. 2). Because of the tilt of Earth's magnetic dipole, each balloon traverses L-shells as it drifts at nearly constant geographic latitude (Fig. 3). Since the balloons move slowly $(\sim 10-30 \mathrm{~km} / \mathrm{h})$ relative to the Earth's surface, each payload samples all local times, but when launched 1-2 days apart, provide average separations of 1-2 hours of MLT. Each BARREL payload will remain aloft in the region of interest for 5-10 days, depending on upper level wind speeds. As balloons drift out of the region, new balloons will be launched to maintain the array; a total of 20 pay- 
loads will be launched during each campaign. Two Antarctic stations were chosen as launch sites because of their locations and logistical considerations. The South African Antarctic station SANAE IV is located at $2.8 \mathrm{~W}, 71.7 \mathrm{~S}$, corresponding to IGRF $L=4.4$, and Halley $\mathrm{VI}$ is located at $26.5 \mathrm{~W}, 75.5 \mathrm{~S}$, corresponding to $L=4.5$. Although only slightly higher in $L$-value, Halley Bay is at higher geographic latitude and further west, providing greater coverage at higher $L$-values.

\subsection{Wave-Particle Interactions}

Precipitation into the atmosphere is generally attributed to wave-particle interactions. The condition for gyro-resonance occurs when the Doppler-shifted plasma wave frequency is equal to the particle gyrofrequency,

$$
\omega-k_{\|} v_{\|}=\frac{n \Omega_{e}}{\gamma}
$$

where $\omega$ is the wave frequency, $k_{\|}$and $v_{\|}$are the parallel components of the wave vector and particle velocity respectively, $\Omega_{e}$ is the non-relativistic electron cyclotron frequency, $\gamma$ is the relativistic factor, and $n$ is a positive or negative integer. When this condition is met, energy can be exchanged between the wave and the particle, leading to acceleration or pitch angle scattering. A more detailed review, including a description of the relevant plasma waves, is given by Millan and Thorne (2007).

Not long after their discovery, low energy microbursts were associated with whistlermode chorus (e.g., Rosenberg et al. 1971), and more recently the association was made for relativistic microbursts (Lorentzen et al. 2001b). In order for an electron with energy of order $1 \mathrm{MeV}$ to be in resonance with typically observed wave frequencies, the interaction must either occur at off-equatorial latitudes $\left(\sim 30^{\circ}\right)$ or be a higher order resonance $(n \geq 3)$ (e.g., Lorentzen et al. 2001b). As mentioned previously, the distributions in $L$-value and magnetic local time are strikingly similar for chorus and microbursts. Additionally, Lorentzen et al. (2001b) found that chorus risers, which are whistler mode waves observed to have a frequency rising in time, last for the same length of time as the coincident microbursts, and the occurrence of microbursts and chorus were generally well correlated for the time periods analyzed. However, as the authors point out, "[c]orrelation does not imply causality," and more observations are needed to quantitatively test models. Most theoretical work applies the quasi-linear, bounce-averaged pitch angle diffusion equation (Schulz and Lanzerotti 1974). However, recent observations of microbursts occurring in conjunction with very large amplitude whistler-mode waves suggest that non-linear trapping may play an important role in the interaction (Kersten et al. 2011). Additionally, microbursts occur on the bounce timescale, thus are too fast for bounce-averaging to be appropriate.

Pitch-angle scattering by EMIC waves was also suggested as a primary loss mechanism for relativistic electrons, since observed wave amplitudes exceeding $1 \mathrm{nT}$ imply scattering in the strong diffusion limit (e.g., Summers and Thorne 2003; Albert 2003). A few studies have found correlated ion and electron precipitation, consistent with scattering by EMIC waves (e.g., Bortnik et al. 2006), but the importance of this mechanism has so far not been quantified. EMIC waves can be excited when substorm-injected ions encounter the cold plasmasphere, leading to a temperature anisotropy that is unstable to the generation of the waves. The injected ions drift westward towards dusk, thus there is believed to be some preference for generation of EMIC waves in the dusk sector, although a peak in their occurrence is also observed near noon where the temperature anisotropy is driven by compressions of the magnetopause (Anderson and Hamilton 1993). In addition to wave generation, the interaction 
itself is expected to be more effective from afternoon to dusk where a plasmaspheric plume stretches to high $L$-values (e.g., Goldstein et al. 2004). The high cold plasma density in the plume lowers the minimum resonance energy for the interaction (e.g., Millan and Thorne 2007). The local time of the duskside precipitation, coincident with the location expected for strong interaction between electrons and EMIC waves, prompted further investigation into whether EMIC waves were the scattering mechanism (e.g., Lorentzen et al. 2000). However, quantitative studies have not been carried out to test this hypothesis due to the lack of good wave observations, and the evidence is so far circumstantial.

The BARREL campaigns will be conducted during the RBSP mission, providing a unique opportunity to obtain measurements of relativistic precipitation in conjunction with in situ observations of plasma waves. The combined BARREL-RBSP measurements will be used to directly test models of wave-particle interactions and will quantitatively test whether whistler-mode chorus and EMIC waves are responsible for microburst precipitation and duskside precipitation respectively. This will be crucial for validating the models that will be used to calculate losses based only on in-situ RBSP measurements, for example during times when global precipitation measurements are not available. The array of balloons distributed in local time and $L$-value maximizes the chance for conjunctions with the two RBSP spacecraft. Two balloon campaigns will be conducted, separated in time by one year. This allows for balloon-spacecraft conjunctions at a range of magnetic local times, since the apogee of RBSP orbits precess at roughly $220^{\circ}$ per year. When correlated measurements are obtained, the RBSP measurements will provide wave power, frequency spectrum, background magnetic field, energetic particle energy and pitch angle distributions, and the cold plasma density, all necessary parameters for using as input to wave-particle interaction models. For example, the quasi-linear diffusion rate and precipitating energy spectrum can be predicted and directly compared with the balloon observations of precipitation to determine whether the diffusion model is consistent with the data.

\subsection{Precipitation Loss Rate}

One of the mysteries of radiation belt variability is the differing electron response for geomagnetic storms that otherwise seem very similar (as characterized by the $D_{s t}$ index). A study of 276 storms by Reeves et al. (2003) showed that only $53 \%$ of the storms resulted in an overall increase in the trapped flux over pre-storm levels, while $19 \%$ resulted in a decrease. This suggests that a sensitive balance exists between acceleration and loss processes. Electrons can be lost from the radiation belts very rapidly; large drops in the flux first observed at geosynchronous orbit have been observed down to $L \sim 4$ (e.g., Morley et al. 2010). These rapid electron depletion events may be due to precipitation (Green et al. 2004) or magnetopause losses (e.g., Ohtani et al. 2009; Turner et al. 2012) or a combination of both (e.g., Millan et al. 2010). Attempts to quantify the precipitation loss rate indicate that the loss can be strong enough to empty the radiation belts in a day or less (Lorentzen et al. 2001a; Millan et al. 2002; O'Brien et al. 2004; Selesnick 2006).

BARREL will determine the precipitation loss rate by making measurements over a wide range of local time and $L$-value. During specific relativistic electron events, the loss rate will be compared to changes in the trapped flux as measured by RBSP and other spacecraft, in order to quantify the role of precipitation relative to magnetopause loss.

\subsection{Large-Scale Spatial Structure of Relativistic Precipitation}

We currently know very little about the spatial distribution of energetic precipitation. Contrast this with our knowledge of the spatial and temporal evolution of lower energy auroral 

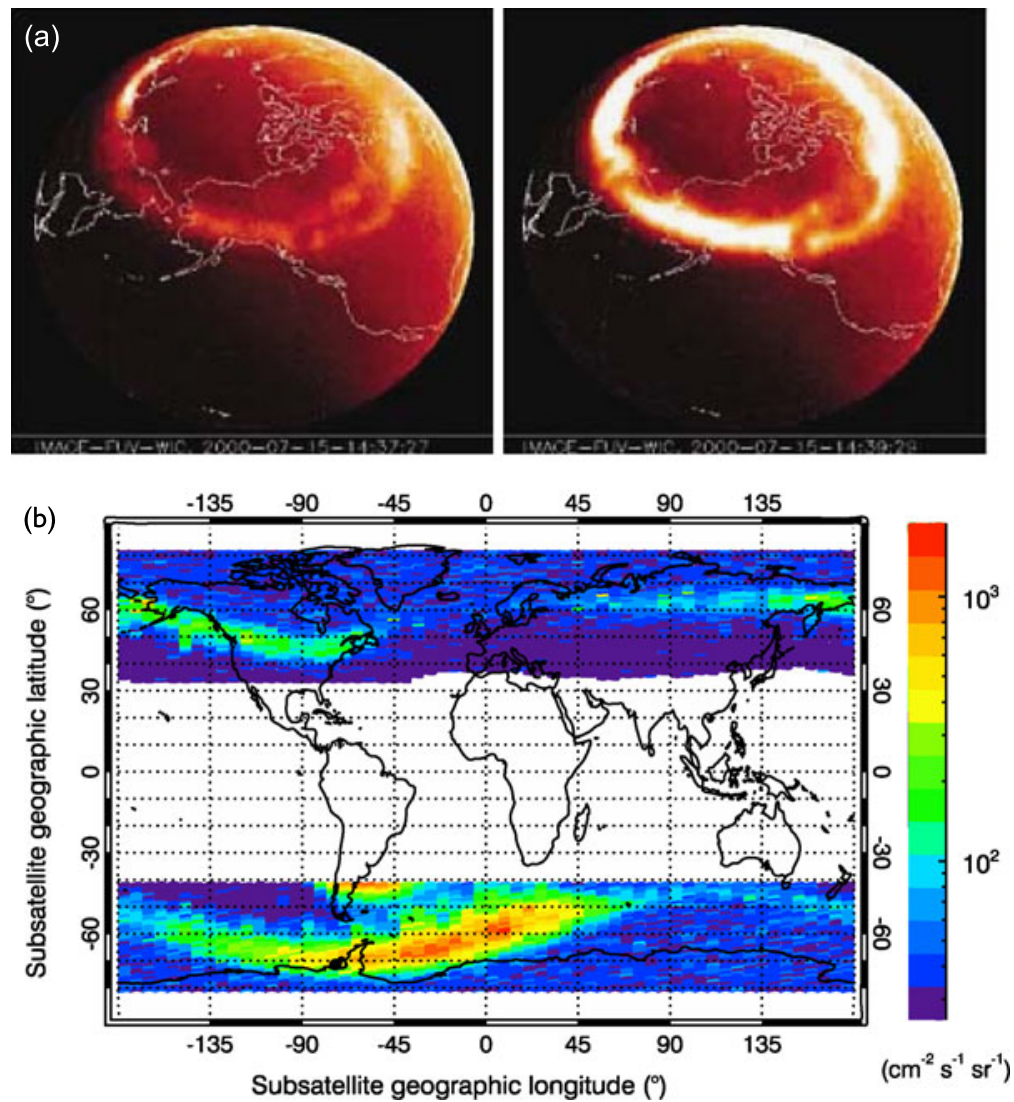

Fig. 4 (a) IMAGE FUV time sequence of the auroral oval on July 15, 2000 (Courtesy NASA/IMAGE FUV team), and (b) Spatial distribution of $>1 \mathrm{MeV}$ precipitating electrons using data from NOAA POES, averaged over the recovery phase of 69 geomagnetic storm, adapted from Horne et al. (2009), Copyright 2009, American Geophysical Union

precipitation, routinely observed with high spatial and temporal resolution as shown in the IMAGE FUV time sequence in Fig. 4a. At relativistic energies, the primary observational tool has so far been single-point spacecraft measurements such as those from NOAA-POES and SAMPEX. These have provided statistical maps of energetic precipitation such as the map of $>1 \mathrm{MeV}$ precipitation shown in Fig. 4b. Such maps reveal important features such as the effects of the South Atlantic Magnetic Anomaly, but the spacecraft revisit a particular location infrequenty, thus limiting the time resolution. Observations from the MINIS balloons revealed complicated temporal and spatial structure of energetic precipitation (Millan 2011) which can only be observed from a nearly stationary platform that does not move quickly through the precipitation region. Untangling the spatial structure of precipitation should reveal information about important boundaries that control or influence precipitation. The spatial scale of the precipitation region is also critical for determining the overall loss rate of electrons discussed in Sect. 2.3.

Another common feature of energetic precipitation is modulation at ULF-timescales, clearly seen in the bottom panel of Fig. 1. Several ideas have been proposed to explain this modulation, but the mechanism is still unknown. It is not clear whether the ULF waves simply modulate the precipitation or whether they play a critical role in causing the scattering. 
ULF waves could be modulating the growth rate of waves, which has been proposed to explain Pc1 pearls through several mechanisms (e.g., Demekhov 2007). Alternatively, if EMIC waves are the cause of the precipitation, modulation of the background magnetic field would lead to a modulation of the minimum resonance energy. For a very steeply falling electron energy spectrum, this would lead to a significant modulation of the precipitating flux. A third suggestion is that the azimuthal electric field of a poloidal mode standing wave may violate an electron's second adiabatic invariant, leading to a decrease in pitch angle for those particles decelerated by the wave in the perpendicular direction (M.K. Hudson, priv. comm.). In each of these cases, one can determine whether the precipitation is modulated in phase or out of phase with the wave magnetic field by comparing measurements of precipitation with both the BARREL magnetic field measurements and those of the RBSP spacecraft.

BARREL will characterize the spatial extent and large scale spatial structure of relativistic precipitation, which has been previously addressed only in a statistical sense. The region over which waves scatter electrons is a critical parameter for modeling electron loss timescales. This is particularly important when direct precipitation measurements are not available. BARREL will simultaneously measure precipitation at about 5 different locations. The data can also be combined with other space and ground assets to produce global maps of precipitation. The nearly-stationary balloon platform will allow for separation of temporal and spatial features, and the study of modulation that is so frequently observed. In particular, the multi-point measurements will allow us to determine the azimuthal mode number of the ULF waves responsible for the modulation. Comparison with RBSP and other spacecraft data will determine whether the modulation is, for example, in phase with the electric or magnetic field, allowing us to constrain the mechanism.

\section{Science Instruments}

\subsection{X-Ray Spectrometer}

BARREL measures relativistic electron precipitation by detecting the bremsstrahlung $\mathrm{X}$-rays produced when electrons collide with atmospheric neutrals. This technique has been used for observing lower energy auroral precipitation for many years (e.g., Winckler et al. 1958; Parks et al. 1993), including the discovery of microburst precipitation (Anderson and Milton 1964). More recently this technique has proved useful for detecting relativistic precipitation (e.g., Foat et al. 1998; Millan et al. 2002). Since relativistic electrons produce $\mathrm{X}$-rays with energies as high as the parent electron energy, the detectors must be efficient at stopping high energy (up to several $\mathrm{MeV}$ ) photons. The BARREL spectrometer system is shown in a block diagram (Fig. 5) with specifications outlined in Table 2, and described in more detail below. The spectral inversion process used to obtain flux and energy information about the parent electrons from the X-ray measurements is discussed in Sect. 6.2.2.

\subsubsection{Instrument Description}

Each BARREL payload includes a single 3 in high $\times 3$ in diameter NaI scintillator which provides a good compromise between energy resolution, detector efficiency (stopping power) and cost. The scintillator crystal is coupled to a photomultiplier tube (PMT), followed by signal processing electronics. The scintillator crystal, the PMT, a high voltage converter and a preamplifier, are all housed in a light-tight aluminum tube (Fig. 6). The assembly is wrapped with a layer of mu-metal shielding to prevent Earth's magnetic field from modulating the PMT gain as the balloon payload rotates or swings. 
Fig. 5 Block diagram of the BARREL X-ray spectrometer

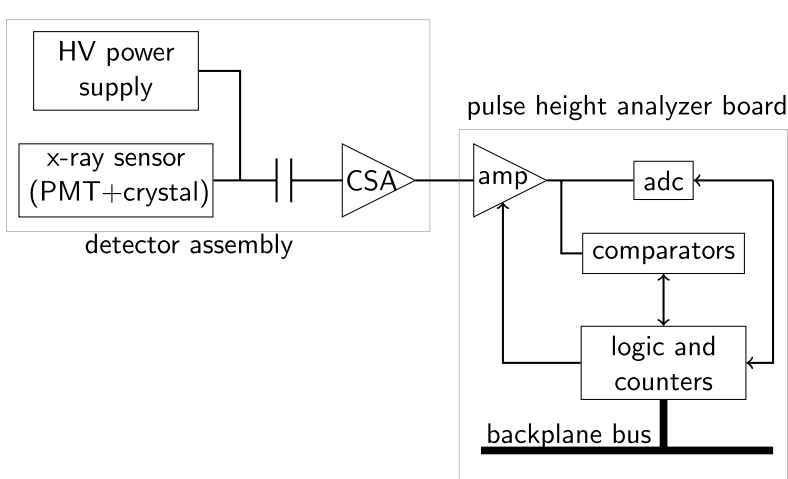

Table 2 Spectrometer specifications

\begin{tabular}{lll}
\hline Attribute & Value & Comments \\
\hline Mass & $2.8 \mathrm{~kg}$ & includes insulation and harness \\
Energy range & $20 \mathrm{keV}-10 \mathrm{MeV}$ & \\
Electronic resolution & $2.4 \mathrm{keV} / \mathrm{channel}$ & reduced by binning \\
System resolution & $7 \%$ at $662 \mathrm{keV}$ & \\
Effective Area & $16 \mathrm{~cm}^{2}$ at $1 \mathrm{MeV}$ & photopeak \\
Dead time per event & $52 \mu \mathrm{s}$ & \\
Operating temperature & $-10{ }^{\circ} \mathrm{C}-40{ }^{\circ} \mathrm{C}$ & efficiency decreases below $\sim 15^{\circ} \mathrm{C}$ \\
Voltage requirement & $\pm 5 \mathrm{~V}$ & \\
Current requirement & $\sim 40 \mathrm{~mA}$ on plus; $\sim 15 \mathrm{~mA}$ on minus & depends on count rate \\
\hline
\end{tabular}

Fig. 6 Cutaway drawing (left) and photo (right) of the detector assembly
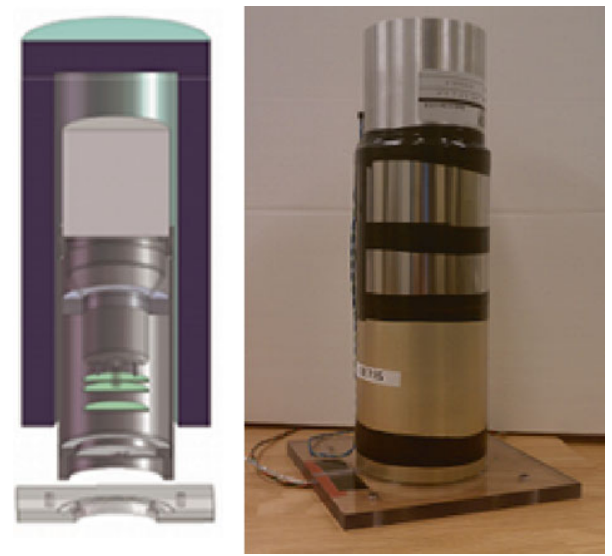

X-rays deposit energy in the scintillator crystal, with a fraction of that energy appearing as a visible light pulse. The PMT converts the light pulse proportionally into a charge pulse, and a charge-sensitive amplifier converts the charge into a voltage pulse. A pulse analyzer board accepts the preamp output and outputs a digital word, whose value is linearly related to the original X-ray energy. The pulse analyzer board contains analog signal processing circuits, an analog to digital converter (ADC), event selection logic, counters, and interface 
logic. Data from the pulse analyzer board is read into the instrument controller board (DPU) described in Sect. 4.1 below.

The BARREL X-ray spectrometer assembly (including scintillator, photomultiplier, signal-processing electronics, and high voltage power supply) is fabricated at the University of Washington and is identical to that flown during the 2005 MINIS balloon campaign, and more recently during the BARREL test flights. The spectrometer assembly design has over 2000 instrument hours of operation at balloon altitude.

\subsubsection{Effective Area and Field of View}

The effective area of the BARREL spectrometer is $16 \mathrm{~cm}^{2}$ (full energy deposited) or $35 \mathrm{~cm}^{2}$ (any fraction of the energy deposited) at $1 \mathrm{MeV}$. The spectrometer is essentially uncollimated and accepts photons over $4 \pi$ steradians. However, the field of view is effectively limited by atmospheric absorption at large angles; Monte Carlo simulations using GEANT3 indicate that the instrument is sensitive to electron precipitation over a $55-60^{\circ}$ cone, corresponding to a $200 \mathrm{~km}$ circle at an altitude of $70 \mathrm{~km}$, typical of where the bremsstrahlung at these energies is created. The spectrometer is oriented such that the field of view is centered on the zenith, and the effects of the balloon in the field of view are negligible since the X-rays easily pass through the very thin $(0.3 \mathrm{mil})$ plastic. Using simulations of the response of $\mathrm{NaI}$ detectors to incoming gamma rays, and of the conversion efficiency of electrons to gammas by the atmosphere, we estimate that the BARREL 3 in $\times 3$ in detectors have an equivalent area/angle product to a perfectly efficient relativistic electron detector, in orbit, of $3 \mathrm{~cm}^{2}$-sr. This is, for example, over twice the factor of the energetic particle detector on the DEMETER spacecraft, although much smaller than the extremely large HILT detectors on SAMPEX. This sensitivity assumes that the precipitation occurs over the entire field of view.

\subsubsection{Energy Resolution}

The spectrometer resolution required to measure and invert the continuum bremsstrahlung spectrum produced by precipitating electrons is about $10 \%$ FWHM at $1 \mathrm{MeV}$. This requirement is met with the readily available standard 3 in $\times 3$ in NaI scintillator described above. The intrinsic energy resolution is measured to be $7 \%$ at $662 \mathrm{keV}$. Resolution and linearity are shown in Fig. 7, an X-ray spectrum of uranium ore. Spectral data are binned to coarser resolution for telemetering as described in Sect. 3.1.5 below.

Fig. 7 Energy spectrum of uranium ore calibration source showing intrinsic spectrometer resolution

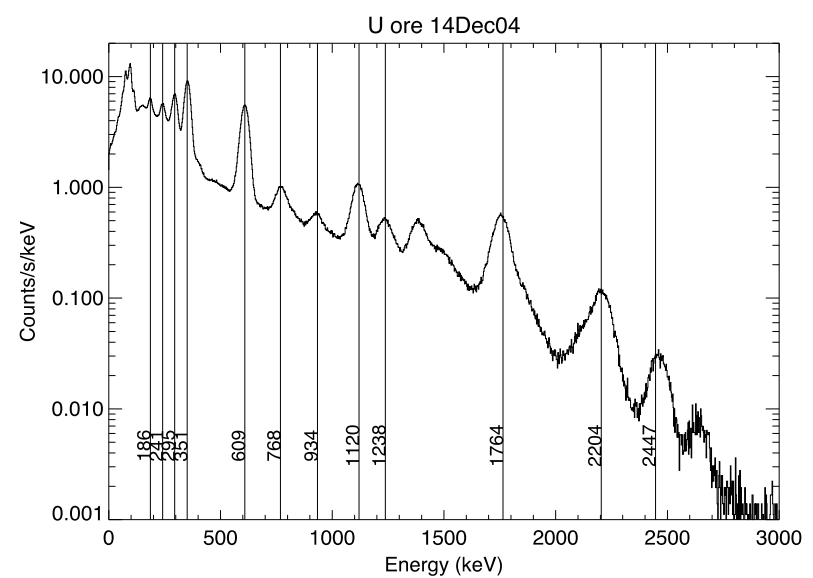




\subsubsection{Time Resolution and Maximum Count Rate}

The time required to analyze a single detector event depends on shaping time constants and the analog to digital conversion time, which is $2 \mu \mathrm{s}$. The analysis time determines the system dead time and maximum allowable counting rate. The total dead time is nominally $5 \mu \mathrm{s}$, corresponding to a few times $10^{5} \mathrm{~s}^{-1}$ peak counting rate. The expected maximum count rate based on previous observations is a few times $10^{4} \mathrm{~s}^{-1}$, well within the instrument capabilities.

Because the arrival time of photons follows Poisson statistics, a small fraction of X-rays are missed due to a small time separation. Correcting for instrument dead time is a wellunderstood procedure (e.g., Knoll 1989). Pulse pile-up can also occur where two pulses are added together into a single large pulse. When this happens, low energy events are undercounted and high energy events are overcounted. However, the time window for this to occur is very small. The instrument electronics are designed such that, once a peak has been detected, an analog switch disconnects the shaping amplifier from the peak-holding capacitor. This prevents a second event from piling onto an earlier event, unless the second event arrives early enough to obscure the peak of the first event.

The spectrometer produces several data products with differing time resolution as described in the next section. The highest time resolution is $50 \mathrm{~ms}$, sufficient for resolving microbursts which have typical durations of $250 \mathrm{~ms}$.

\subsubsection{Spectrometer Data}

The spectrometer produces two kinds of data products: rate counters for monitoring instrument health, and digitized energies of individual X-ray photons, which are further binned into fast-, medium-, and slow-rate spectra before being telemetered. The rate counters include lower and upper discriminator counters, an interrupt counter, and a valid peak detect counter. Each of these count over a four second interval and is telemetered once every four seconds.

$\mathrm{X}$-ray photons arrive at random times and must be read on demand. This is accomplished by having the spectrometer interrupt the DSP when it has acquired an X-ray. Individual X-ray events are binned into three spectral products (Table 3) which trade between energy and time resolution. As shown in Fig. 8, fast spectra are binned into four broad energy channels at $50 \mathrm{~ms}$, providing the highest time resolution, but it should be noted that the energy ranges will shift with instrument temperature due to gain changes.

Medium-rate spectra are accumulated every four seconds into 48 bins spanning an energy range of approximately $100-4000 \mathrm{keV}$. Slow spectra covering the full energy range are accumulated into 256 bins every 32 seconds. Slow spectra bin widths increase geometrically from roughly $2.4 \mathrm{keV} / \mathrm{bin}$ (first $64 \mathrm{bins}$ ), to $154 \mathrm{keV} /$ bin (last $32 \mathrm{bins}$ ). These bin widths will also shift with temperature, a fact especially important when accumulating spectra over long

Table 3 Spectrometer data products

\begin{tabular}{llll}
\hline Product & Cadence $(\mathrm{s})$ & Energy Range $(\mathrm{keV})$ & \# Energy Channels \\
\hline Rate Counters & 4 & - & - \\
Fast Spectra & 0.05 & $20-1500$ & 4 \\
Medium Spectra & 4 & $100-4000$ & 48 \\
Slow Spectra & 32 & $20-10000$ & 256 \\
\hline
\end{tabular}


Fig. 8 Example of fast spectra taken during the 2008/2009 BARREL Antarctic test flight, adapted from Millan (2011).

$\mathrm{X}$-ray count rate is shown in four energy channels corresponding to $<180 \mathrm{keV}$ (blue), 180-550 keV (red), 550-840 keV (green), and 840-1500 keV (yellow)

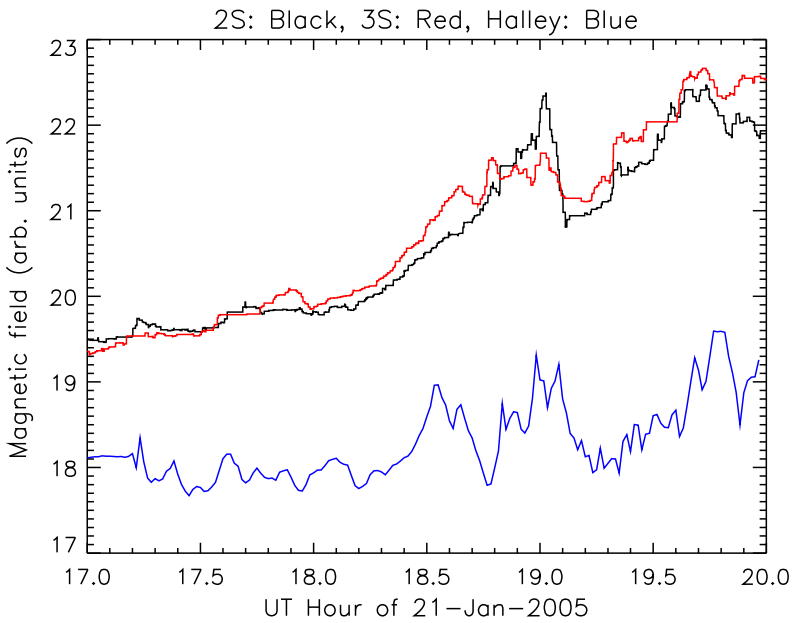

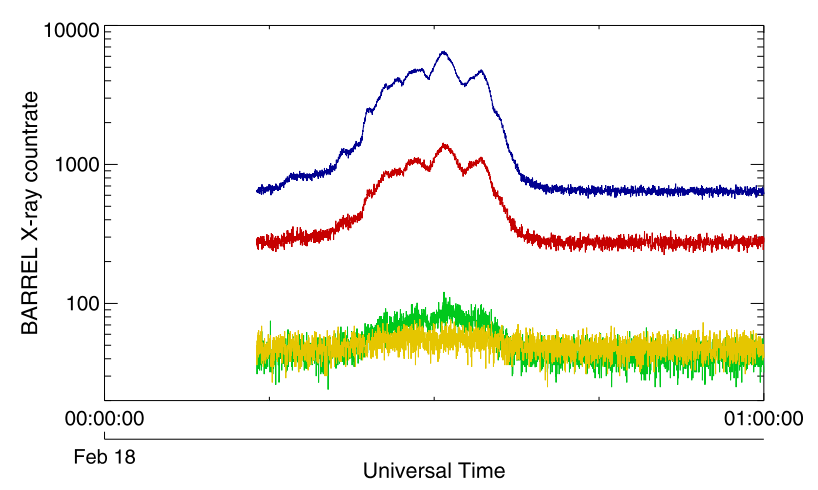

Fig. 9 Smoothed DC Magnetometer data taken showing total magnetic field during 2005 MINIS flights 2/South and 3/South (red and black) along with data from Halley Bay (blue) 
Fig. 10 Bartington Mag648

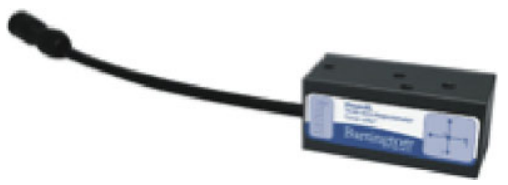

\subsubsection{Sensor}

BARREL uses a Bartington Mag648 Series three-axis fluxgate magnetometer with full scale range of $\pm 100 \mu \mathrm{T}$ (Fig. 10), read out as three balanced differential outputs over 0 to $\pm 3 \mathrm{~V}$. The manufacturer's specification for the sensor internal noise level ranges between 10-20 $\mathrm{pT}_{r m s} / \sqrt{\mathrm{Hz}} @ 1 \mathrm{~Hz}$.

\subsubsection{Electronics}

The three analog signals from the Bartington sensor are sampled at $4 \mathrm{~Hz}$ by a 24-bit, 4channel analog-to-digital converter (Analog Devices AD7193). The ADC includes a lowpass digital filter with a sinc $\mathrm{c}^{4}$ shape and $40 \mathrm{~ms}$ settling time. The sequencing of measurements from each of the three axes is handled within the chip. Each output consists of the 24-bit conversion (Data Register) and 12-bits of information about the conversion (Status Register) creating a 36-bit word per channel. The status register contains useful information for processing such as the channel that supplied the data. The magnetometer data are passed to the BARREL Data Processing Unit (DPU) via an RS-232 serial interface consisting of an 8-bit microcontroller (Microchip Technology PIC16F84A) and driver (Analog Devices ADM3222).

\subsubsection{Performance}

The ultimate noise performance of our magnetometer system (including the electronics) has not been reached either in laboratory tests or test flights due to magnetic noise from laboratory electronics and the payload. The lowest noise figure in our best-controlled environmental test has been $0.1 \mathrm{nT} \mathrm{rms} \mathrm{in} \mathrm{the} \mathrm{final} 4 \mathrm{~Hz}$ data. In test flight conditions, the noise per axis has varied from approximately 0.2 to $3.0 \mathrm{nT} \mathrm{rms}$, due to currents in the payload. A variety of boom configurations were tested to minimize this noise, subject to the constraint that the boom doesn't make it difficult to balance the payload. The system gain has a small temperature dependence that varies from unit to unit and axis to axis. This gain drift is measured by placing the magnetometer in a thermal chamber that is surrounded by a large three-axis Helmholtz coil.

\subsubsection{Magnetometer Data Products}

The raw (Level 0) magnetometer data consist of three orthogonal magnetic field values every 0.25 seconds. Because of the orientation of the sensor relative to the payload, what the sensor reports as $B_{y}$ corresponds to the vertical magnetic field (positive upwards), positive $B_{z}$ is along the boom, outward from the payload, and $B_{x}$ is perpendicular to $B_{y}$ and $B_{z}$, all measured relative to the payload. Practially speaking, the payload exhibits pendulum motions, thus it is necessary to transform the data into a geophysical coordinate system. Since we have no independent azimuthal aspect sensor, the data products that will be of most use will be $B_{\text {vert }}$ and $B_{\text {horiz }}$; these will be the primary scientific product, although users will have access to the raw $B_{x}, B_{y}, B_{z}$ values, as measured in the payload coordinate system. 
Fig. 11 Data taken during 2010 BARREL test flight: two horizontal components of the magnetic field in $\mu \mathrm{T}$ (top), the derived walk in angle (middle), and the angular speed (bottom)
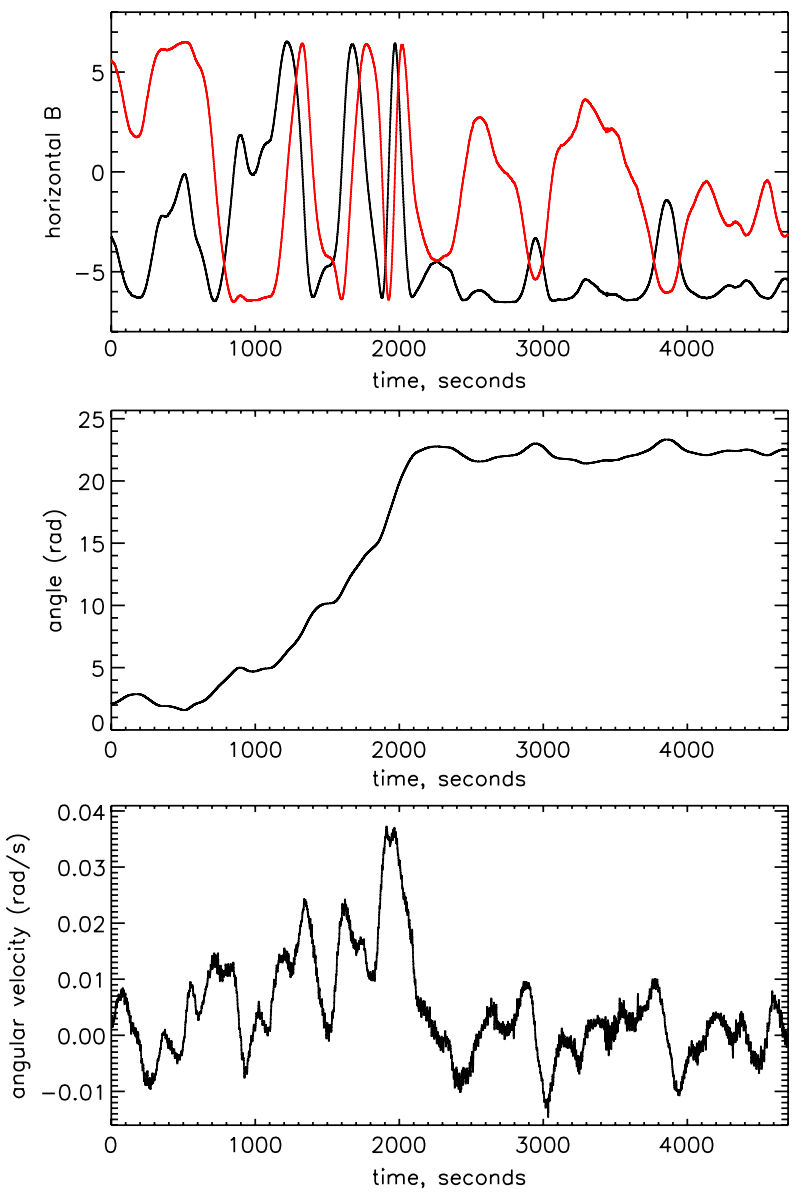

\subsubsection{Interpretation of Magnetometer Data}

Since the BARREL payloads are freely rotating and swinging, both of these motions will appear in the raw data. The torsional behavior at float is a sort of smoothed random walk in angle, presumably driven by horizontal wind gradients at the balloon, creating torsion transmitted through the load line to the payload. For the most part, this input is slow compared to the natural torsional oscillation period of the balloon on the load line, so periodic oscillations are not expected to be prominent. Figure 11 shows an interval of data from a 2010 BARREL test flight. From top to bottom, the plots are of the two horizontal components of the field, the derived walk in angle, and the angular speed. We will use closer analysis of such data to identify the torsional and pendulum frequencies of the payload so that they are not mistaken for waves in the magnetic field. Our analytical estimates of the periods are approximately 5 seconds for simple pendulum motion and 40 seconds for torsional motion.

While the temperature variation of gain in each axis of each magnetometer will be known from ground calibration, other parameters of the magnetometers can only be determined to sufficient precision using in-flight data. These are the tilt of the vertical magnetometer axis from true vertical, non-orthogonalities among the three axes (expected to be less than 0.5 degrees according to the manufacturer's specification), constant DC offsets in each axis (due 
to both the payload's field and the ADC), and differences in gain among the three axes at fixed temperature. The random motions of the payload during times of relatively constant temperature (noon and midnight) will allow us to define these parameters for each payload by forcing the derived $B_{z}$ and $B_{\text {horiz }}$ to be nearly constant and consistent with geomagnetic models. The calibration will be done using data during geomagnetically quiet times, so that the resulting parameters can be applied to times when the field itself is not expected to be constant.

\section{Payload Design and Support Systems}

\subsection{Data Processing Unit}

\subsubsection{Instrument Controller Board}

The BARREL DPU acquires data from the X-ray spectrometer, magnetometer, a GPS module, and an engineering data interface (EDI). It outputs formatted data frames to an Iridium modem for downlink, and controls the command-cutdown system. The DPU is a general purpose instrument controller board, which communicates with other boards through a backplane and a memory-type interface. Flexibility is achieved through the use of an FPGA that contains interface logic for the backplane signals. The instrument controller board has flown successfully on three sounding rocket experiments, and about 26 balloon flights, most recently in the 2010 BARREL test flights.

\subsubsection{Navigation and Timing}

Time and positioning are provided by a GPS module, model Lassen SQ manufactured by Trimble, which resides in the DPU box. Each second, GPS time and position data are passed to the DPU through a backplane-connected UART. In addition, the GPS module emits an accurate timing pulse, which signals the DPU to save a free-running $1 \mathrm{kHz}$ clock. By saving this clock value on each GPS second, and injecting GPS second of the week into the telemetry stream, the payload clock is synchronized to GPS time with $1 \mathrm{~ms}$ accuracy. Regular synchronization is necessary because the DPU's crystal oscillator undergoes a temperature drift.

\subsubsection{Engineering Data}

The engineering data interface (EDI) performs analog to digital conversion of key temperatures, voltages, and currents. There are 8 inputs of voltage and current pairs, 4 inputs of voltage only and 16 inputs nominally for temperature sensors. Temperature inputs can also be configured to monitor voltage. All inputs are scaled to the nominal $5 \mathrm{~V}$ ADC full-scale range and converted 2 channels at a time with 16-bit precision.

Each of the 8 voltage/current inputs has a voltage divider to scale the input and a differential current-sense amplifier to measure the voltage across an external resistor in series with the current flow. Two inputs employ inverting amplifiers for sensing voltage/current from negative supplies. The 4 voltage-only inputs employ a voltage divider to scale the input. Thirteen of the sixteen temperature sense inputs have a resistor to $5 \mathrm{~V}$ to supply approximately $1 \mathrm{~mA}$ of current to LM335 temperature sensors, which provide $10 \mathrm{mV} /{ }^{\circ} \mathrm{K}$ output. Three remaining temperature inputs are configured as voltage inputs. All inputs are referenced directly to DPU ground. All housekeeping output digital words are linearly related to physical units such as temperature, voltage, or current. 


\subsubsection{Flight Software}

The BARREL DPU contains a digital signal processor (ADSP-2101) which runs the flight software, and a boot flash memory chip to store the flight software when power is off. The BARREL software is a simple real-time operating system. The main functions are to control and read data from several peripheral devices, format the data into frames, and emit a stream of data frames to the telemetry system.

The software consists of two stages. The first stage initializes memory (RAM) and configuration registers, the internal clock, and the UART and peripheral devices. The second stage is the main software loop, in which the DPU sleeps until interrupted. On an interrupt, the DPU services the interrupt, traverses the main loop, checking on events flagged for attention, and then goes back to sleep. Scheduled interrupts arrive every millisecond, generated by an internal timer. Asynchronous interrupts arrive mostly from the X-ray spectrometer (1000's per second), upon acquisition of an X-ray. For each X-ray, interrupt handling software increments a bin in the accumulating slow, medium, and fast spectra. The minimum time between two X-ray interrupts, including the signal processing and analog to digital conversion time, is about $6 \mu \mathrm{s}$. The interrupt service time is from 1.1 to $4.2 \mu \mathrm{s}$, with higher energy photons taking more time to bin. The GPS interrupts at one second marks, to provide an accurate timing pulse.

With regard to telemetry and command, the DPU software has two functions: (1) produce data frames and send them to the modem; (2) receive and execute commands from the ground. Ground software is described in Sect. 6 below. Flight software constructs data frames which are accumulated in a toggling buffer pair and transmitted at the rate of one frame per second (currently 214 bytes/second). Housekeeping data, including the EDI measurements, as well as additional GPS data (GPS week, number of satellites, UTC seconds offset), and miscellaneous information (number of dropped calls, number of modem resets, command counter) is sub-commutated on a 40-frame cycle. Four commands are recognized: three for the cutdown system (arm, disarm, cut), and a no-op command. The ARM command stores energy in capacitors which will supply high current to the termination squib. The DISARM command drains the stored energy through resistors. The CUTDOWN command activates a relay to fire a squib and terminate the flight.

\subsection{Power System}

The BARREL power system is based on a solar-charged nickel metal hydride (NiMH) battery that powers a box of DC/DC converters which provide isolated regulated voltages to payload components. The use of solar power is typical for long duration balloon flights in the polar regions which experience 24 hours of sunlight. The power system was developed for BARREL, based on the same solar panels that CSBF uses, and on a UC Berkeley flightheritage charge controller (from the HIREGS LDB payload) and DC/DC converters. The only significant change is the choice of battery chemistry. BARREL uses NiMH batteries, chosen for having a higher capacity-to-weight ratio than lead-acid and for its greater robustness in comparison to lithium-based systems in response to charge cycles over a wide range of temperatures. Each BARREL payload requires less than $5 \mathrm{~W}$ average total power. A summary block diagram of the complete power system is shown in Fig. 12, followed by a more detailed specification of the individual power system components.

\subsubsection{Solar Panels}

The BARREL payloads are freely spinning, thus the power system relies on 4 identical solar panels, one for each side of the payload. In addition to satisfying the payload power 


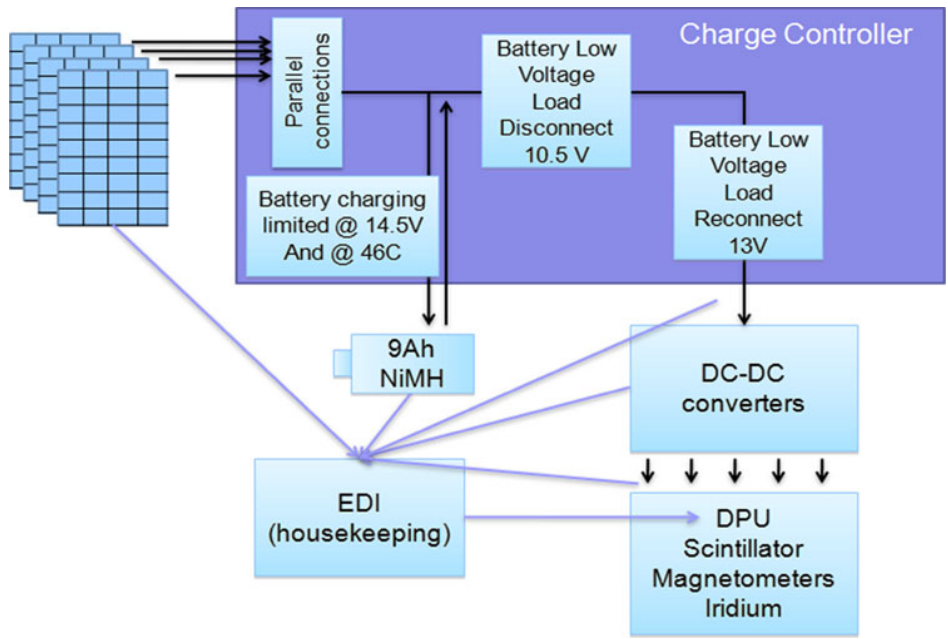

Fig. 12 Power system block diagram

requirements at any orientation to the sun, this provides for some redundancy should a panel be damaged during launch.

BARREL uses custom-built solar panels (Suncat Solar, LLC) to ensure sufficient panel voltage at the high panel temperatures experienced in the balloon environment (up to $100{ }^{\circ} \mathrm{C}$ ). The four BARREL panels consist of a laminated honeycomb structure $16.25^{\prime \prime} \times$ $21.5^{\prime \prime} \times 0.5^{\prime \prime}$ with 40 series-connected Sunpower cells which have been cut to the minimum size allowed by the all-back-contact cells $\left(1.5^{\prime \prime} \times 4.875^{\prime \prime}\right)$. Each panel is $\sim 400 \mathrm{~g}$ without mounting structure. Expected performance is $V_{m p}$ of $19 \mathrm{~V}$ at $25^{\circ} \mathrm{C}$. Based on flight data from the BARREL test flights, we expect $\sim 2.5$ A from any panel that is close to fully illuminated.

\subsubsection{Charge Controller and Battery}

The BARREL charge controller is designed to accept 4 parallel solar panel inputs and maintain a battery voltage between 10.5 and 14.5 V. Fundamentally it is a pair of switches (implemented with a pair of IRF5305 FETs), that (1) connect the load when the battery voltage is high enough, and (2) disconnect the solar panels if the battery voltage is approaching its maximum. Hysteresis is present on both switches to avoid oscillation. Panel power is used at whatever current the panels can supply whenever the panel voltage is above the battery voltage (minus a small FET and Shottky diode drop) up to a maximum voltage of $36 \mathrm{~V}$ (LM293 limits). When total panel current is above the payload requirements, excess current is stored in battery charge up to the point where the battery is fully charged. When panel current is below the payload required current, the battery makes up the deficit by discharging to its minimum allowed voltage. A thermistor is also included in the battery pack which allows the charge controller to cut battery charging at $46{ }^{\circ} \mathrm{C}$ and resume below $42{ }^{\circ} \mathrm{C}$. A backup snap action bimetal cutoff is also present in the battery packs with a $60^{\circ} \mathrm{C}$ trip point. This simple charge/discharge arrangement is suitable for nickel chemistry rechargeable batteries with small internal resistances and large capacity.

The battery itself is made of 10 series connected GP9000 NiMH cells (rated at $9 \mathrm{~A}$ h) with nominal voltage of $12 \mathrm{~V}$ and a range from $10.5-14.5 \mathrm{~V}$. The payload requires $\sim 500 \mathrm{~mA}$, 
which implies about $2 / 3$ of a day in maximum battery capacity, or with derating, about 12 hours. Under typical operations (in full sun), the battery generally remains at full charge, but the excess battery capacity allows for continued operation if sunset is experienced late in the season.

In addition to maintaining battery charge the charge controller outputs to the EDI 6 voltages and 2 currents (measured as voltages across series resistance of $0.025 \Omega$ ) for housekeeping purposes.

\subsubsection{DC/DC Converter Box}

Each DC/DC converter accepts 9-18 V DC from a power supply, the BARREL charge controller, or a battery, and outputs voltages for the rest of the payload. In addition to supplying payload power, the DC/DC converter box outputs voltages and currents to the EDI (measured as voltages across series resistance $100 \mathrm{mV}$ full scale) for housekeeping purposes. DC/DC conversion is accomplished using the isolated CALEX 12s5.1000HW with moderate filtering. The Iridium modem has its own internal DC/DC converter and accepts a wider range of voltages than the rest of the BARREL payload. The DC/DC converter box passes its input voltage to the Iridium after minimal filtering and a current sense resistor.

\subsection{Telecommunications}

Long-duration balloon flights require either data storage or an over-the-horizon telemetry system for real-time communications, since the payload generally moves out of line-ofsight range within about one day. In the case of BARREL flights from Antarctica, recovery of payloads may be difficult due to the remote location. Therefore, a data storage system is not a practical nor reliable way to retrieve data; a real-time telemetry system is thus required.

The BARREL telemetry needs are primarily driven by the required energy and time resolution of X-ray data. The baseline telemetry requirement for BARREL is a data rate of 214 bytes/s which can be achieved using the Iridium satellite network. Iridium provides real-time communications at up to $\sim 3 \mathrm{kbits} / \mathrm{s}$ with both downlink and uplink capabilities. BARREL uses the Iridium Satellite Modem Model A3LA-X from NAL Research, weighing $340 \mathrm{~g}(0.75 \mathrm{lb})$ and drawing an average of $800 \mathrm{~mA}$ at $5.0 \mathrm{~V}$. The modem operates with the Iridium network at $1616-1626.5 \mathrm{MHz}$ and interfaces with the DPU via serial RS-232 using standard modem AT commands. The flight modem operates in auto-answer mode; if the connection is lost, a new call is automatically initiated by the ground station which includes an identical modem. Flight data are not saved in memory when a call is dropped; the reconnect time is typically 30 seconds or less, based on test-flight data. In the event that the flight modem doesn't recognize a lost connection, the DPU can do a soft modem reset (after 1 hour), or power cycle the modem (after 4.5 hours).

\subsection{Flight Train and Termination System}

Each payload is carried to a float altitude of about $37 \mathrm{~km}$ by an Aerostar Model SF-0.300.3/0-TA zero-pressure helium-filled balloon. Due to the remote launch site and need for launching payloads at a rate of about one every two days, this balloon size was chosen because it is "hand-launchable" (Fig. 13). The 0.3 mil-thick 300,000 cubic ft. balloon is made of polyethylene and can carry a maximum payload weight of 72 pounds. The payload is suspended from the balloon by the flight train, which includes an $8 \mathrm{ft}$ diameter parachute and a $16 \mathrm{ft}$ long ladder made from $4800 \mathrm{lb}$ nylon rope. Termination of each flight is achieved 
Fig. 13 Balloon launch over

Halley VI Research station

during 2013 BARREL Campaign

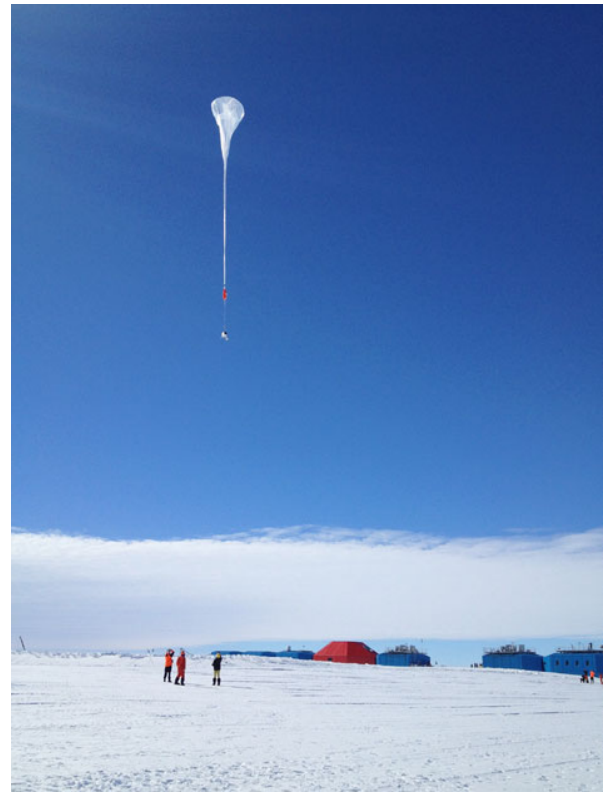

with a small explosive guillotine cutter, fired by the terminate system which is suspended at the apex of the parachute. The terminate can be commanded from the ground through the Iridium telemetry system or autonomously in the event that the payload altitude drops below 60,000 feet and contact with the ground has been lost.

\subsection{Mechanical Design}

The BARREL mechanical design was largely driven by the requirement to fabricate 45 payloads quickly and at low cost. The structure must also be as light as possible, but strong enough to survive chute-shock, and must provide easy access to each subsystem. In order to meet an ambitious operations schedule of one launch every two days, the payloads are shipped fully assembled, with the exception of the science instruments and solar panels which are attached at the launch site. The payload design consists of an internal frame constructed of aluminum angle, a foam enclosure, and an external aluminum frame for attaching the flight train and solar panels and to allow for easy launch. Handles made of square rails allow for easy transport and launch. Improvements to the design were made primarily to the external frame after the first BARREL test campaign. These allowed for a simplification of the manufacturing process and improved accessibility to the instrument subsystems while at the launch site.

\subsubsection{Internal Structure}

The internal frame is $23^{\prime \prime}$ tall and just over $8^{\prime \prime}$ wide and is made of four aluminum angle "legs" attached with aluminum angle cross-bars. The scintillator is housed in the middle of the frame and sits in a plexi-glass flange that is bolted to a $0.08^{\prime \prime}$ thick aluminum plate. The electronics boxes are mounted to each side of the frame (Fig. 14a), and the battery sits beneath the payload. Cable harnessing is secured to the frame with cable ties. The frame slips into a $2^{\prime \prime}$ thick foam enclosure for passive thermal control (described below). Cut-outs 

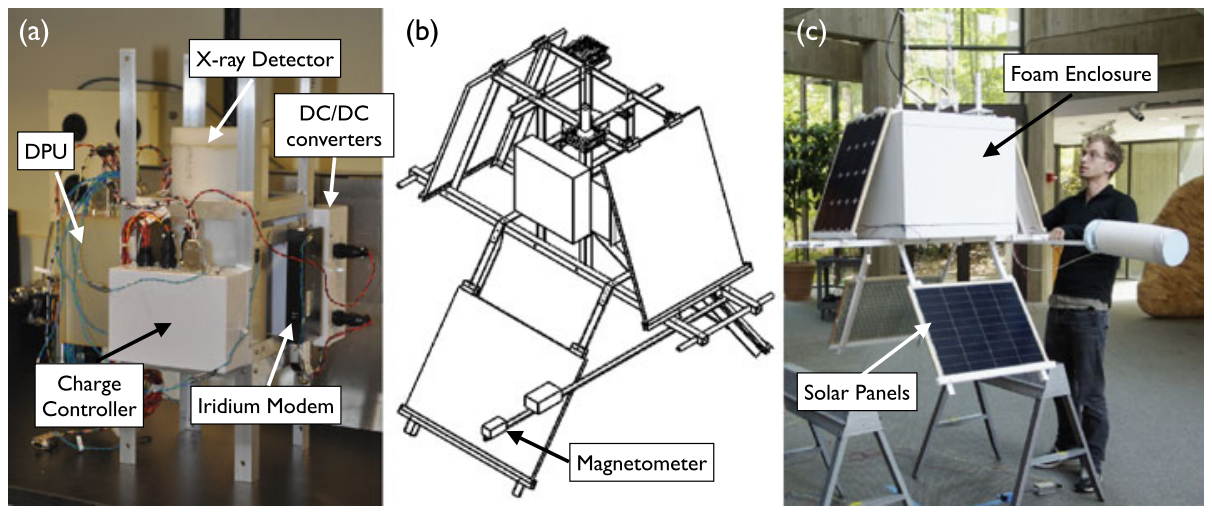

Fig. 14 (a) Internal payload structure, (b) mechanical cutaway drawing, and (c) fully assembled payload, including foam enclosure and solar panels

in the foam allow the GPS antenna cable, Iridium antenna cable, magnetometer serial cable, and solar panel cables to run to the outside.

\subsubsection{External Structure}

Figure 14b shows the external frame attached to the internal structure. Two $50^{\prime \prime}$ long aluminum square rails run parallel beneath the internal structure outside the foam enclosure and are attached to the internal structure legs with $1 / 4^{\prime \prime}$ bolts. A $1 / 2^{\prime \prime}$ diameter aluminum tube passes through the square rails on each side, serving as handles to carry and launch the payload. The square rails support the $42^{\prime \prime}$ magnetometer boom as well as the aluminum $\mathrm{H}$-channel used to suspend the solar panels, two above the square rails on opposite sides of the payload and two below the payload on the other sides. On top of the payload, outside the foam enclosure, a "tic-tac-toe"-shaped structure is attached to the four payload legs and allows for mounting of the Iridium and GPS antennas. This structure also aids in keeping the upper solar panels held in place. The fully assembled payload, including foam thermal enclosures and solar panels is shown in Fig. 14c.

\subsubsection{Manufacturing}

Manufacturing of mechanical parts was primarily carried out by students at Dartmouth College with the oversight and assistance of the professional machinists. A series of jigs-one jig for each unique part-was fabricated in order to streamline and insure precision in the drilling of holes. This allowed for an assembly-line approach whereby different people could reliably perform the same tasks in a repeatable way. Parts were machined and painted en masse, and then assembled as needed.

\subsection{Thermal Control}

Balloon payloads are subjected to temperatures ranging from very cold $\left(-50{ }^{\circ} \mathrm{C}\right)$ on ascent through the tropopause to very hot $\left(+50{ }^{\circ} \mathrm{C}\right)$ at float altitude due to a lack of convection at those altitudes and the high level of sunlight reflected off the snow/ice surface of Antarctica. Thermal control of balloon payloads can usually be achieved with simple passive measures such as foam thermal enclosures, thermal coupling of components, and appropriate choice 
of surface properties (e.g., aluminized mylar or white paint) since thermal energy transfer is generally dominated by radiation. The majority of BARREL subsystems have temperature operating ranges of or exceeding $-40^{\circ}$ to $+60^{\circ} \mathrm{C}$. The thermal design is primarily driven by the scintillator which requires a thermal rate of change less than $8^{\circ} \mathrm{C}$ per hour (to prevent the crystal from cracking) and preferred temperature above $0{ }^{\circ} \mathrm{C}$ to maintain the instrument efficiency.

Thermal design was aided by the Thermal Desktop package, which is an overlay module to AutoCAD, performing radiative and conductive heat transfer calculations. The thermal model was validated during the BARREL test flights in 2009 and 2010. Each BARREL payload is enclosed in a $2^{\prime \prime}$ thick foam box which is painted white (Fig. 14b). All exterior metal surfaces are also painted white to reflect sunlight. To reduce its rate of temperature change, the scintillator is conductively isolated from the rest of the payload structure using a plexiglass mounting plate, and is surrounded by an additional inch of foam. The magnetometer is also insulated with $1^{\prime \prime}$ of white foam since it is mounted on an exterior boom. Two of the four solar panels hang below the payload where they can radiate to open space rather than the main payload, thus reducing the internal payload temperatures.

\section{Instrument Calibration}

\subsection{X-Ray Spectrometer}

For the spectrometer, the most important calibration is the energy scale. The standard ${ }^{137} \mathrm{Cs}$ $662 \mathrm{keV}$ line is used to set the PMT nominal operating high voltage and overall gain. After making this adjustment, spectra are collected from background and several radioactive sources to provide a set of energy calibration points. The gain, which determines the relation between channel number and energy bin, depends on temperature, and has been characterized between $5{ }^{\circ} \mathrm{C}$ and $30{ }^{\circ} \mathrm{C}$ in the laboratory. From these line measurements, a gain model is constructed, and the overall system energy resolution obtained. Because the relation between channel and energy is not quite linear, multiple lines are needed to characterize it in the laboratory. Because the form of the nonlinearity is predictable and reproducible for a given scintillator crystal design, once it has been measured using a number of lines, a single measurement of a single line is enough to establish the entire channel-energy relation to better than $5 \%$. During flight, the $511 \mathrm{keV}$ annihilation line produced by cosmic rays, is readily observable and usable for in-flight calibration. Atmospheric gamma lines from ${ }^{14} \mathrm{~N}$ can also have sufficient intensity during intense precipitation events or solar proton events. Temperature measurements in flight provide a cross-check by giving an independent means to estimate which channel the $511 \mathrm{keV}$ line should occupy.

Measurements of the dead-time response of the detectors at extremely high count rates are made using bright radioactive sources at gradually decreasing (and carefully measured) distance from the detectors. GEANT3 simulations are used to predict what rates the detectors should be seeing and will be made to agree with the data at large distances, where detector dead-time is negligible, by adjusting the source strength in the simulation. The ratio between the predicted and observed count rates as they start to saturate at high rates will be recorded and used to correct observed count rates in flight should a particularly bright precipitation event (or solar flare, or solar particle event) occur. For most of the duskside relativistic electron precipitation and relativistic microburst events that make up the primary BARREL science, this dead-time correction should be minor or negligible. 


\subsection{Magnetometer}

Gain calibration for the magnetometer is carried out at UCSC using a Helmholtz coil surrounding a thermal chamber. A chopped current put through the coil allows the gain to be measured with a known signal, even when the background field in the laboratory is changing. Data are collected in $10^{\circ}-20^{\circ}$ increments, between $-40^{\circ}$ and $+50^{\circ} \mathrm{C}$ while chopping the Helmholtz field amplitude between $25-50 \mu \mathrm{T}$. The thermal chamber is turned off during data collection, after the desired temperature is reached. From the data, a gain calibration parameter is determined for each axis. A typical value for the gain drift with temperature is $0.02 \%$ per degree C. The sense is usually toward increasing gain with increasing temperature, although some axes reverse this trend. During flight, a temperature sensor is mounted to the case of the magnetometer. The temperature sensor is itself calibrated during I\&T. Gain corrections can then be applied in post-processing.

The magnetometer is mounted to a $42^{\prime \prime}$ long boom to reduce the effects of stray currents in the payload, particularly the varying currents of the solar panels. Residual effects of payload currents are measured during an outdoor/full-sun Comprehensive Performance Test. In a separate test, the payload is suspended from the entire flight train and magnetometer data are analyzed to determine the payload natural pendulum frequencies. However, it is anticipated that in-flight data will provide the best characterization of payload motions.

\subsection{Clock Synchronization}

Since each BARREL payload carries a GPS clock, time synchronization should be straightforward. Test flights have shown tracking of GPS time to better than $10 \mathrm{~ms}$ using only the time stamp that is transmitted at a 4 second cadence. Nevertheless, it is important to verify timing between payloads, because multi-point measurements are critical to BARREL science. To verify the synchronization at a sub-second level, the following test is carried out in the laboratory. A set of four operating payloads is exposed to a simultaneous impulsive radiation signal by rapidly moving the cover of a bright radioactive source that illuminates the payloads. The data are recorded and analyzed at UCSC MOC to verify that the event appears simultaneous in all payloads to better than $0.25 \mathrm{~s}$.

\section{Operations and Data Analysis}

\subsection{Mission Operations and Ground Systems}

The BARREL ground segment is managed by University of California, Santa Cruz. During normal operations, Iridium downlink is received at the UCSC Mission Operations Center (MOC). Backup downlink capabilities are located at U.C. Berkeley and Dartmouth College.

A schematic of the BARREL ground systems is shown in Fig. 15. The MOC hardware consists of a collection of five laptop computers running Windows XP. Four of the computers are configured to run up to four instances of the ground station software simultaneously, and one computer serves as a backup. Four Iridium modems (NAL research model AL3A$\mathrm{X}$ ), are connected to each of the primary computers via a 4-channel serial/ USB converter, supporting satellite communication to four payloads per computer. Each modem is connected via an RF cable to an Iridium antenna (NAL research model SAF5350-CX) bolted to a fixture on the roof of the Natural Sciences II building at UCSC. MOC computers are all networked to a common storage device, the MOCs network attached storage (MOC-NAS). 


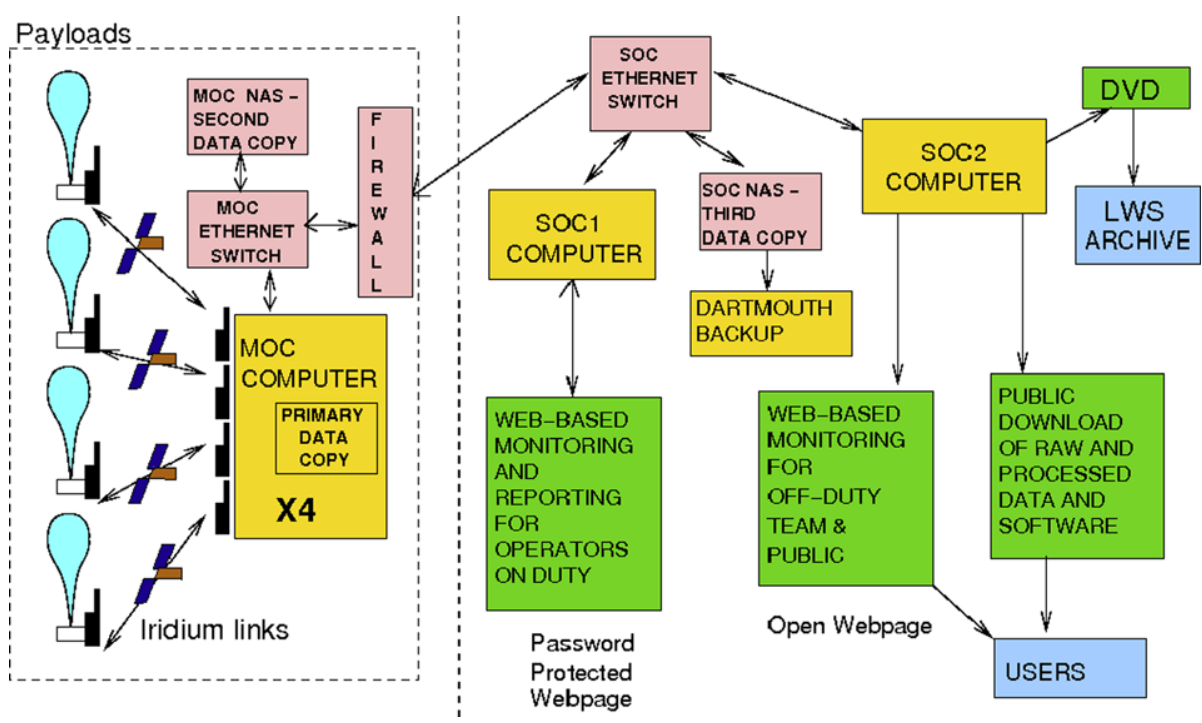

Fig. 15 Schematic of BARREL MOC/SOC

The connection is made through an Ethernet switch which also provides a connection to the UCSC network via a hardware firewall. The only connection allowed through the firewall is for the Science Operations Computer, SOC1, (see below) to read data from the MOCNAS. The MOC hardware (MOC-NAS, switch, firewall, laptops, and Iridium modems) are powered through a UPS sized for at least 3 hours during an outage.

The GSE software on the MOC computers is $\mathrm{C}$ code written in the LabWindows environment, and an associated user interface for displaying science and housekeeping data. The code is a significantly revised version of the GSE software used during the MINIS 2005 balloon campaign. Each instance of the software supports a single flight payload. The software auto-detects when new data are not being received and automatically initiates a new Iridium call to the payload. A binary data file is opened locally and on the NAS for each new Iridium call that is initiated. Raw data frames are written to this file as received, with no further processing by the MOC. Although the flights will be monitored continuously, the software is designed to operate autonomously and very little human intervention is required.

Flight monitoring and science operations are handled by two computers separate from the MOC (SOC1 and SOC2). An Ethernet switch connects SOC1, SOC2, and the SOC-NAS to the UCSC network. SOC1 and SOC2 are server-style tower computers running Linux. SOC1 is primarily used to process the data found on the MOC-NAS and store it on the SOC-NAS. SOC1 also serves a special, password-protected version of the web interface that allows some administrative control. The web server responsible for the bulk of web traffic is hosted on SOC2. This separation ensures that excessive web traffic will not interfere with processing real-time data.

The BARREL SOC software package is used to collect, archive, and disseminate the information from the MOC-NAS raw data files. Local and remote users can see a live feed of the raw data files as they are written and use a number of visualization tools to analyze past data (Fig. 16). This is done by providing three main functions: near real-time conversion of incoming binary data files into ASCII and CDF archive files; data monitoring and text message notification of values that exceed maximum limits; and a web interface for viewing 


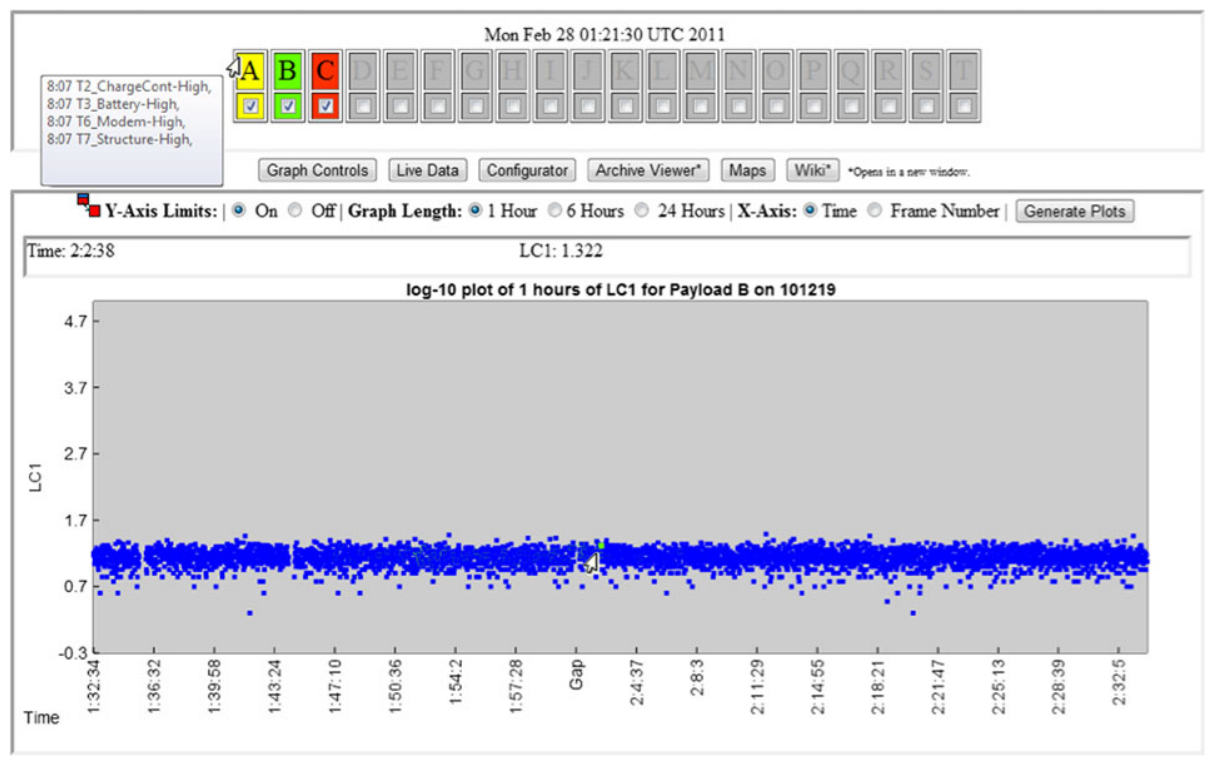

Fig. 16 Screenshot of SOC web interface

live and archived data in a number of different ways. Data products are described in more detail in Sect. 6.2 below.

Mission operations are staffed 24/7, with one mission monitor working a three-hour shift at any given time. Monitoring is carried out via the web interface to SOC1, which allows for monitoring by geographically distributed team members. A report is generated on a team wiki site by the mission monitor at the end of each shift. The mission monitor also notifies a duty scientist in the event of any payload or GSE anomalies. SOC2 is available for data viewing by other team members or the public.

\subsection{Science Data Products and Analysis}

\subsubsection{Data Products}

BARREL summary data will be made available to the public in near real-time. Summary plots and a map of payload locations can also be viewed from the BARREL SOC website during the BARREL flights. Archived data will be released as Level 0-3 products about 24-48 hours after collection, summarized in Table 4. The Level 0 data product is a cleaned version of the raw binary data (i.e. with duplicate or corrupt frames removed). One Level 0 data file will be produced for each payload each day. Level 1 data are CDF files of timetagged uncalibrated data. Calibrated data will be released as Level 2 files in CDF and in IDLSAVE formats. These include energy-scale calibrated X-ray spectra, magnetometer data, ephemeris files, and other housekeeping information. Higher level (Level 3) data products may be released for specific event intervals. For current information about data products and analysis tools, visit the BARREL project website (http://www.dartmouth.edu/ barrel).

\subsubsection{Data Analysis}

In addition to CDF data files, analysis tools written in the IDL language will be made available through the BARREL SOC website. These will include basic plotting tools used to 
Table 4 BARREL Data Products

\begin{tabular}{lll}
\hline Level & Description & Format \\
\hline L0 & TM file (1/payload/day), good frames only & binary (BARREL) \\
L1 & Time-tagged, uncalibrated (e.g., counts/s) & CDF \\
L2 & Calibrated (physical units) & CDF, IDLSAVE \\
L3 & Higher level products (e.g., spectrograms) & CDF \\
\hline
\end{tabular}

display X-ray count rates and magnetic field data versus time, routines for creating Fourier power spectrograms, and routines for creating X-ray count spectra over specified time intervals.

The conversion between the spectrum of counts observed in balloon-borne X-ray detectors and the spectrum of electrons incident on the atmosphere is a well-established one, and not a new development (e.g., Berger and Seltzer 1972; Foat et al. 1998). Nonetheless, we describe it here because it is the primary BARREL measurement. Monte Carlo simulations using GEANT4 are carried out to simulate the conversion of electrons to detector counts. In a sense, one can consider Earth's atmosphere as part of the instrument, responsible for converting the incident electrons into photons that are detected by the scintillator. Incident precipitating electrons are Coulomb scattered and produce bremsstrahlung X-rays in the atmosphere. The photons are scattered in both the atmosphere and the instrument before ultimately being absorbed (or exiting the detector if not fully absorbed). The effects of Compton scattering, photoelectric absorption, and, at the highest energies, pair production and the production of secondary bremsstrahlung; are included in the simulations. A mass model of the payload was constructed along with a model of the atmosphere. GEANT4 also allows for inclusion of Earth's magnetic field. The entire process is modeled for mono-energetic electrons between $40 \mathrm{keV}-4 \mathrm{MeV}$ to obtain a response matrix which provides detector counts as a function of energy for each incident electron energy and for a given balloon altitude.

Event count spectra are analyzed using a "forward-folding" technique: a model energy spectrum is assumed for the incident electrons, and the known response based on the Monte Carlo simulations is used to predict the expected count spectrum in the detector at its known altitude. The model count spectrum is normalized to minimize chi-square with the data-the normalization then telling us the intensity of the initially assumed electron spectrum. The process can be repeated, varying the parameters and form of the incident electron spectrum until the best fit is obtained and the range of incident spectral parameters that are allowed by the data to a certain level of confidence are determined. Thus, for example, we might conclude that a given precipitating electron spectrum is consistent with an exponential form with folding energies between 600 and $700 \mathrm{keV}$, but not consistent with a power law of any index. The BARREL instrument response matrix will be provided along with tools for using it to invert the X-ray spectrum to obtain information about the precipitating parent electrons.

\section{Summary}

We have discussed known types of relativistic electron precipitation and shown that there are many unanswered questions about the mechanisms causing energetic electron loss. The spatial-temporal evolution of precipitation at relativistic energies is largely unexplored, and the temporal modulation of precipitation at ULF timescales that has been observed routinely remains unexplained. Finally, the relative importance of losses to the atmosphere versus losses to the magnetopause is not currently known. To address all of these questions, 
the BARREL Investigation will consist of two balloon campaigns carried out in January 2013 and 2014, during the RBSP mission. The combination of in situ RBSP measurements and low altitude BARREL measurements of precipitation provides a unique opportunity to quantitatively test models of wave-particle interactions thought to be responsible for precipitation.

Acknowledgements BARREL is supported by the NASA Living With a Star program through NASA grant NNX08AM58G at Dartmouth College. Support for operations was provided by the Columbia Scientific Balloon Facility, the NASA Balloon Program Office, and the National Science Foundation's Office of Polar Programs. MAC has received funding from the Natural Environmental Research Council under the Antarctic Funding Initiative (AFI/11/22). The BARREL science campaigns were supported by NERC/British Antarctic Survey, SANSA Space Science, and the University of KwaZulu-Natal.

Open Access This article is distributed under the terms of the Creative Commons Attribution License which permits any use, distribution, and reproduction in any medium, provided the original author(s) and the source are credited.

\section{References}

J.M. Albert, Evaluation of quasi-linear diffusion coefficients for EMIC waves in a multispecies plasma. J. Geophys. Res. 108(A6), 1-9 (2003)

B.J. Anderson, D.C. Hamilton, Electromagnetic ion cyclotron waves stimulated by modest magnetospheric compressions. J. Geophys. Res. 98, 11369 (1993)

K.A. Anderson, D.W. Milton, Balloon observations of X rays in the auroral zone. J. Geophys. Res. 69, 44574479 (1964)

M.J. Berger, S.M. Seltzer, Bremsstrahlung in the atmosphere. J. Atmos. Terr. Phys. 34, 85-108 (1972)

J.B. Blake, M.D. Looper, D.N. Baker, R. Nakamura, B. Klecker, D. Hovestadt, New high temporal and spatial resolution measurements by SAMPEX of the precipitation of relativistic electrons. Adv. Space Res. 18(8), 171-186 (1996)

J. Bortnik, R.M. Thorne, T.P. O'Brien, J.C. Green, R.J. Strangeway, Y.Y. Shprits, D.N. Baker, Observation of two distinct, rapid loss mechanisms during the 20 November 2003 radiation belt dropout event. J. Geophys. Res. 111(A12), 12216 (2006)

C. Cattell, J.R. Wygant, K. Goetz, K. Kersten, P.J. Kellogg, T. von Rosenvinge, S.D. Bale, I. Roth, M. Temerin, M.K. Hudson, R.A. Mewaldt, M. Wiedenbeck, M. Maksimovic, R. Ergun, M. Acuna, C.T. Russell, Discovery of very large amplitude whistler-mode waves in Earth's radiation belts. Geophys. Res. Lett. 35, 01105 (2008)

A. Demekhov, Recent progress in understanding Pc1 pearl formation. J. Atmos. Sol.-Terr. Phys. 69, 1609 (2007)

J.E. Foat, R.P. Lin, D.M. Smith, F. Fenrich, R. Millan, I. Roth, K.R. Lorentzen, M.P. McCarthy, G.K. Parks, J.P. Treilhou, First detection of a terrestrial MeV X-ray burst. Geophys. Res. Lett. 25(22), 4109-4112 (1998)

J. Goldstein, B.R. Sandel, M.F. Thomsen, M. Spasojević, P.H. Reiff, Simultaneous remote sensing and in situ observations of plasmaspheric drainage plumes. J. Geophys. Res. 109(A), 03202 (2004)

J.C. Green, T.G. Onsager, T.P. O'Brien, D.N. Baker, Testing loss mechanisms capable of rapidly depleting relativistic electron flux in the Earth's outer radiation belt. J. Geophys. Res. 109, 12211 (2004)

R.B. Horne, M.M. Lam, J.C. Green, Energetic electron precipitation from the outer radiation belt during geomagnetic storms. Geophys. Res. Lett. 36, 19104 (2009)

W.L. Imhof, H.D. Voss, J. Mobilia, D.W. Datlowe, E.E. Gaines, J.P. McGlennon, U.S. Inan, Relativistic electron microbursts. J. Geophys. Res. 97, 13829 (1992)

K. Kersten, C.A. Cattell, A. Breneman, K. Goetz, P.J. Kellogg, J.R. Wygant, L.B.I. Wilson, J.B. Blake, M.D. Looper, I. Roth, Observation of relativistic electron microbursts in conjunction with intense radiation belt whistler-mode waves. Geophys. Res. Lett. 38, 08107 (2011)

G.F. Knoll, Radiation Detection and Measurement, 2nd edn. (Wiley, New York, 1989)

M. Kokorowski, E.A. Bering, M. Ruohoniemi, J.G. Sample, R.H. Holzworth, S.D. Bale, J.B. Blake, A.B. Collier, A.R.W. Hughes, E.H. Lay, R.P. Lin, M.P. McCarthy, R.M. Millan, H. Moraal, T.P. O’Brien, G.K. Parks, M. Pulupa, B.D. Reddell, D.M. Smith, P.H. Stoker, L. Woodger, Magnetospheric electric field variations caused by storm-time shock fronts. Adv. Space Res. 42, 181 (2008) 
K.R. Lorentzen, M.D. Looper, J.B. Blake, Relativistic electron microbursts during the GEM storms. Geophys. Res. Lett. 28(1), 2573-2576 (2001a)

K.R. Lorentzen, M.P. McCarthy, G.K. Parks, J.E. Foat, R.M. Millan, D.M. Smith, R.P. Lin, J.P. Treilhou, Precipitation of relativistic electrons by interaction with electromagnetic ion cyclotron waves. J. Geophys. Res. 105(A), 5381-5390 (2000)

K.R. Lorentzen, J.B. Blake, U.S. Inan, J. Bortnik, Observations of relativistic electron microbursts in association with VLF chorus. J. Geophys. Res. 106(A), 6017-6028 (2001b)

L.R. Lyons, R.M. Thorne, Equilibrium structure of radiation belt electrons. J. Geophys. Res. 78, 2142-2149 (1973)

R.M. Millan, Understanding relativistic electron losses with BARREL. J. Atmos. Sol. Terr. Phys. (2011)

R.M. Millan, R.M. Thorne, Review of radiation belt relativistic electron losses. J. Atmos. Sol.-Terr. Phys. 69(3), 362-377 (2007)

R.M. Millan, R.P. Lin, D.M. Smith, K.R. Lorentzen, M.P. McCarthy, X-ray observations of MeV electron precipitation with a balloon-borne germanium spectrometer. Geophys. Res. Lett. 29, 2194 (2002)

R.M. Millan, R.P. Lin, D.M. Smith, M.P. McCarthy, Observation of relativistic electron precipitation during a rapid decrease of trapped relativistic electron flux. Geophys. Res. Lett. 34(10), 1-5 (2007)

R.M. Millan, K.B. Yando, J.C. Green, A.Y. Ukhorskiy, Spatial distribution of relativistic electron precipitation during a radiation belt depletion event. Geophys. Res. Lett. 37(2), 20103 (2010)

S.K. Morley, R.H.W. Friedel, T.E. Cayton, E. Noveroske, A rapid, global and prolonged electron radiation belt dropout observed with the Global Positioning System constellation. Geophys. Res. Lett. 37, 06102 (2010)

R. Nakamura, M. Isowa, Y. Kamide, D.N. Baker, J.B. Blake, M. Looper, SAMPEX observations of precipitation bursts in the outer radiation belt. J. Geophys. Res. 105(A), 15875-15886 (2000)

T.P. O'Brien, M.D. Looper, J.B. Blake, Quantification of relativistic electron microburst losses during the GEM storms. Geophys. Res. Lett. 31(4), 04802 (2004)

S. Ohtani, Y. Miyoshi, H.J. Singer, J.M. Weygand, On the loss of relativistic electrons at geosynchronous altitude: its dependence on magnetic configurations and external conditions. J. Geophys. Res. 114, 01202 (2009)

G.K. Parks, T.J. Freeman, M.P. McCarthy, S. Werden, The discovery of auroral X-rays by balloon-borne detectors and their contributions to magnetospheric research, in Auroral Plasma Dynamics. Geophysical Monograph, vol. 80, (American Geophysical Union, Washington, D.C., 1993), pp. 17-23

G.D. Reeves, K.L. McAdams, R.H.W. Friedel, T.P. O’Brien, Acceleration and loss of relativistic electrons during geomagnetic storms. Geophys. Res. Lett. 30, 36 (2003)

T.J. Rosenberg, R.A. Helliwell, J.P. Katsufrakis, Electron precipitation associated with discrete very low frequency emissions. J. Geophys. Res. 76, 8445 (1971)

M. Schulz, L.J. Lanzerotti, Particle Diffusion in the Radiation Belts. Springer, Heidelberg (1974)

R.S. Selesnick, Source and loss rates of radiation belt relativistic electrons during magnetic storms. J. Geophys. Res. 111(A), 04210 (2006)

D. Summers, R.M. Thorne, Relativistic electron pitch-angle scattering by electromagnetic ion cyclotron waves during geomagnetic storms. J. Geophys. Res. 108, 1143 (2003). doi:10.1029/200JA009489

R.M. Thorne, C.F. Kennel, Relativistic electron precipitation during magnetic storm main phase. J. Geophys. Res. 76, 4446-4453 (1971)

R.M. Thorne, Radiation belt dynamics: the importance of wave-particle interactions. Geophys. Res. Lett. 37, 22107 (2010)

D.L. Turner, Y. Shprits, M. Hartinger, V. Angelopoulos, Explaining sudden losses of outer radiation belt electrons during geomagnetic storms (2012)

A.Y. Ukhorskiy, B.J. Anderson, P.C. Brandt, N.A. Tsyganenko, Storm time evolution of the outer radiation belt: transport and losses. J. Geophys. Res. 111, A11S03 (2006)

M. Walt, W.M. MacDonald, Diffusion of electrons in the van Allen radiation belt, 1, Treatment of particles with mirroring points at high altitude. J. Geophys. Res. 67, 5013-5024 (1962)

J.R. Winckler, L. Person, R. Arnoldy, R. Hoffman, X-rays from visible aurora at Minneapolis. Phys. Rev. 110, 1221 (1958) 